\title{
Nitrosamine (and /or Losartan/ Hydrochlorothiazide) Induced Pretibial Located Lentigo Maligna: First Reported Case in the World Literature
}

\author{
Tchernev $\mathbf{G}^{1}$, Oliveira $\mathbf{N}^{1}$, Kandathil $\mathbf{L J}^{1}$, Milev $\mathbf{S}^{2}$ \\ ${ }^{1}$ Onkoderma - Clinic for Dermatology, Venereology and Dermatologic Surgery, Sofia, Bulgaria \\ ${ }^{2}$ MBAL SKIN SYSTEMS, Doganovo, Bulgaria
}

Received: August 03, 2021; Accepted: August 15, 2021; Published: August 18, 2021

*Corresponding author: GeorgiTchernev, Onkoderma, Clinic for Dermatology, Venereology and Dermatologic Surgery, General Skobelev, , Email: georgi_tchernev@yahoo.de

A 66-year-old Caucasian woman presented to the dermatology department with complaints of a pigmented lesion on the left shin. She first noticed the lesion in March 2020, several months prior to presentation but decided to seek medical advice after observing substantial growth in the size and shape of the lesion. She was diagnosed with type II arterial hypertension 6 years ago and was started losartan/hydrochlorothiazide $50 \mathrm{mg} / 12.5 \mathrm{mg}$ half a tablet daily. The patient visits her general practitioner regularly and her hypertension is well controlled on these medications, had no other concomitant diseases and was relatively healthy for her age. No family history for dermatologic diseases was available. On dermatologic examination, a single superficially spreading pretibial lesion covering $1.8 \mathrm{~cm}$ wide and $1.2 \mathrm{~cm}$ high was noted(Figure 1a). The light greyish-pink pigmented lesion was oval in shape with sharply demarcated borders and no elevation above the surrounding skin. Further laboratory work-up showed no signs of deviations. Based on the clinical and dermatoscopic evidence, the diagnosis of melanoma in situ, lentigo maligna type was made. She was sent for treatment at a specialised hospital for oncological diseases in June 2020 where a surgical excision was performed under local anaesthesia with $0.5 \mathrm{~cm}$ clear/ negative margins in all directions. The defect was thereafter closed using a full-thickness mesh graft taken from the abdominal region(Figure 1b). The excised tumour was subsequently sent for histological evaluation and was keeping with the clinicopathological diagnosis of lentigo maligna. Immunohistochemical analysis also identified S100 positive nests of cells within the basal lamina, confirming the diagnosis.Postsurgical wound healing with the skin graft was poor due to inadequate graft transplantation whereby necrotic as well as macerated areas were observed leadingto bad cosmetic results (Figure 1c-h).After a multidisciplinary review and follow up, a second operation was performed a month later to remove the necrotic, together with the hypertrophic tissue and a new split skin mesh graft was utilised. Stringent post-operative care, including prophylactic antibiotic therapy was also provided to promote good wound healing which was achieved several months later (Figure 1i).

The potentialrole of nitrosamines in the treatment of hypertension with angiotensin receptor blockers/ sartans has been repeatedly discussed and identified as possible independent factor for melanoma development and possible progression [1,2].In-dept analyses and follow-up of serious international retrospective studies in this direction strongly confirm the significant dependence between the intake of sartans / nitrosamines and the development of melanomas subsequently[3,4]. It should be noted that at the same time $(2015 / 2016)$ the role of nitrosamines was not the subject of discussion and investigation $[3,4]$.

The case we share is the first in the world literature regarding the development of melanoma precursor lesion in the form of lentigo maligna after starting losartan/ Nitrosamine (?) therapy for arterial hypertension.

The open questions/ dilemmas facing clinicians, independent controlling authorities/ drug agencies and researchers are numerous, given the following facts:

1. One of the few serious first in vitro studies available according the role of losartan / with probable available carcinogenic impurities in the form of nitrosamines? / as a possible potent potentiating agent for melanoma cell metastasis has been already published also in 2018 [5]?

2. Another important in vitro study shares similar experimental data on the possible procarcinogenic effect of losartan in vitro / again it is unclear whether it is not the effect of nitrosamines as possible impurities? /and dates from 2019 (6).In both mentioned studies there is no data whether the active substance of losartan used in vitro is nitrosamine free $[5,6]$.

3. The introduction of permissible doses of nitrosamines in sartans turns out to be an indisputable fact, confirmed by the EMA [7]?

4. International scientific data show that the optimal methodology for quantification of nitrosamines in medicine is still lacking [8].

5. Many open questions about the Nitrosamine role in the pathogenesis of various oncological skin diseases remain without a definitive decision till the present moment and the 

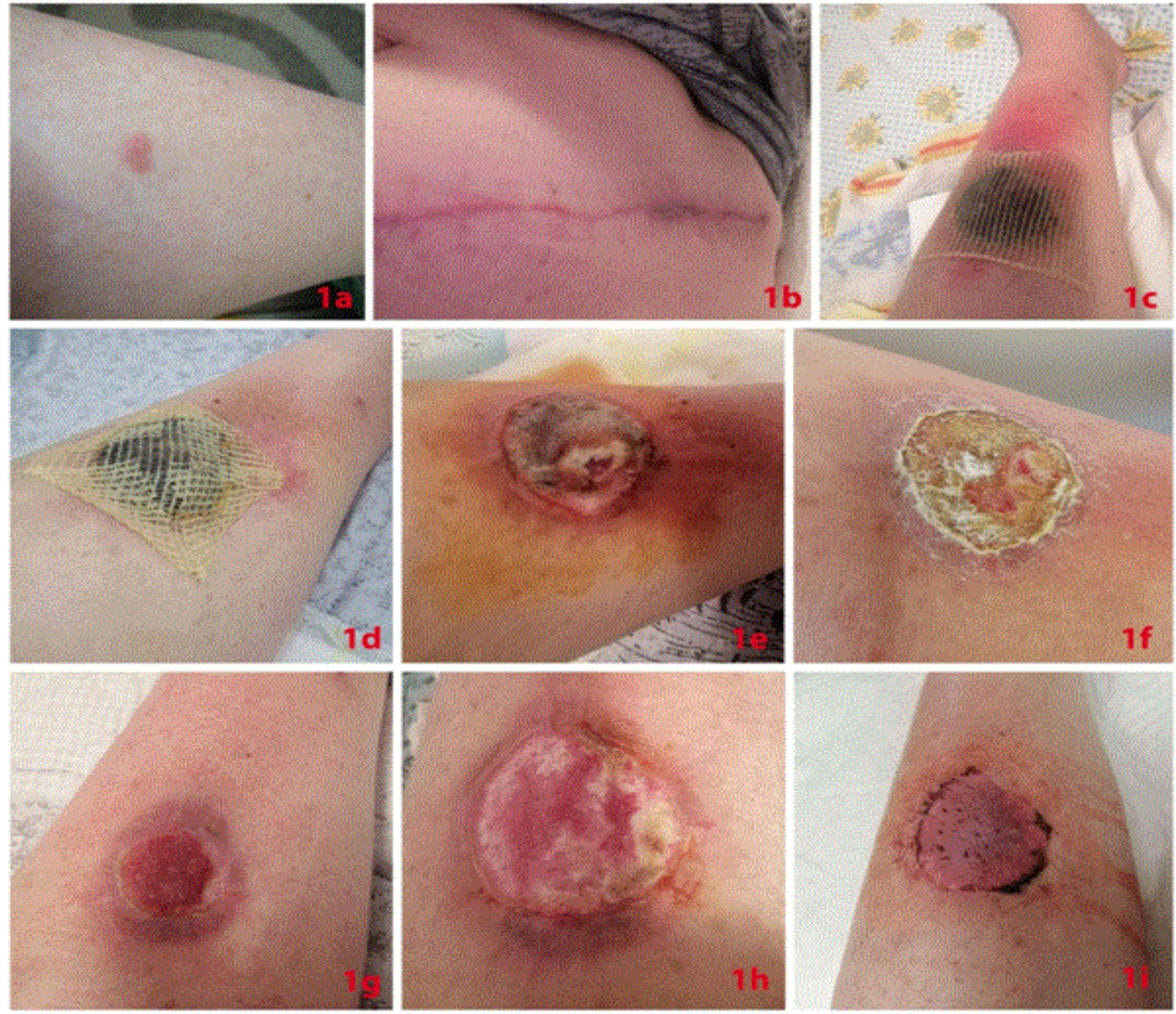

Figure 1a: Pretibially located lentigo maligna like lesion as observed clinically and dermoscopically.

Figure 1b: Abdominal area after completed full thickness mesh graft attempted which was unsuccessful and with unsatisfactory cosmetic results.

Figure 1c/1d: Direct postoperative results after the first operative session with some necrotic, macerated skin areas, directly after the performance of full thickness mesh graft.

Figure 1e/1f: Poor healing after the first surgical session.

Figure 1g/1 h: Hypertrophic areas within the resected tissue after removal of the poorly healed transplanted tissue.

Figure 1i: Clinical picture after second surgical session and new split skin mesh graft with good healing tendency several months later.

controversy opinons shared by the regulatory authorities seems to be problematic for clinicians and patients.

The fact that monotherapy with thiazide diuretics, such as hydrochlorothiazide, for example, is associated with a significantly higher risk of developing melanoma than monotherapy with sartans is further problematic [4]. The combination of sartans with thiazide diuretics is likely to multiply this risk [2].

The possible presence of nitrosamines in sartans is thought to have a synergistic effect on the development of melanomas, similar (but pathogenetically different) to the effect of thiazide diuretics [2]. It is because of these facts that the combination of both groups of medicaments (Sartans/ thiazides) could be defined as "double trouble" [2].

The lack of possibility/ deliberate non-implementation and subsequent formal disclosure of checks for the presence/ absence of nitrosamines in certain geographical areas (even in Europe) certainly makes it difficult to clarify a number of dilemmas such as: nitrosamines in sartans and/ or thiazides are the leading link in the processes of triggering the carcinogenesis. The fact that a number of clinical observations confirm the simultaneous manifestation of melanoma in combination with other tumours definitely suggest a synergistic but at the same time different action of these substances within the carcinogenesis. Thanks.

\section{References}

1. Tchernev G, Bitolska A, Patterson JW. Telmisartan (and/or nitrosamine) - induced occult melanoma: first reported case in world literature. Expert Rev Clin Pharmacol. 2021;10:1-6. doi: 10.1080/17512433.2021.1938547.

2. Tchernev G, Patterson JW. Telmisartan/hydrochlorothiazide-induced nevus-associated cutaneous melanoma: first report in the medical literature. Expert Rev Clin Pharmacol. 2021;14(3):289-293. doi: 
10.1080/17512433.2021.1890581.

3. Schmidt SA, Schmidt M, Mehnert F, Lemeshow S, Sorensen HT. Use of antihypertensive drugs and risk of skin cancer. J Eur Acad Dermatol Venereol. 2015;29(8):1545-1554. doi: 10.1111/jdv.12921.

4. Sable K, Majewski S, Nardone B, Cices A, West DP, Laumann AE. Association of melanoma and nonmelanoma skin cancer with antihypertensive drugs: A report from the Research on Adverse Drug events and Reports project. Poster 3167. J Am Acad Dermatol 2016;74(5):AB221.

5. Olschewski DN, Hofschroer V, Nielsen N, Seidler DG, Schwab A, Stock C. The Angiotensin II Type 1 Receptor Antagonist Losartan Affects NHE1-Dependent Melanoma Cell Behavior. Cell Physiol Biochem. 2018;45(6):2560-2576.
6. Renziehausen A, Wang H, Rao B, Weir L, Nigro CL, Lattanzio L, et al. The renin angiotensin system (RAS) mediates bifunctional growth regulation in melanoma and is a novel target for therapeutic intervention. Oncogene. 2019;38(13):2320-2336.

7. Sartans medicinal Products: EMA`s Assesment Report defined Nitrosamine Limits and reporting.

8. Johnson GE, Dobo K, Gollapudi B, Harvey J, Kenny J, Kenyon M, et al. Permitted daily exposure limits for noteworthy N-nitrosamines. Environ Mol Mutagen. 2021;62(5):293-305. 